\section{Can neutron starlight be seen?}

AMONG the more exotic inhabitants of our Galaxy are the enigmatic neutron stars. Although these objects are about the same mass as ordinary stars, they are only a few kilometres in diameter, and are therefore unbelievably dense. So much so that their constituent atoms are entirely crushed by gravity into neutrons. Despite having been predicted theoretically for many years, firm evidence for their existence had to await the discovery and interpretation in the late 1960s of the now famous pulsars. Pulsars are radio sources which emit bursts of energy at very regular durations, usually about once a second. Current models of these cosmic clocks assume their regular ticking to be caused by the rapid rotation of an extremely compact object coupled to a magnetic field. The compact object is generally thought by astronomers to be the long awaited neutron star, born during the violent aftermath of a supernova explosion.

Models of the pulsar mechanism are nezessarily tentative, but the pulses of radiation are believed to originate not from the neutron star itself, but rather from the surrounding magnetosphere. This then leaves open the question of what radiation might be expected direct from the neutron star surface. The answer to this depends on the temperature of the surface, which is really an unknown factor. General considerations, however, point to this radiation being in the $\mathrm{X}$-ray region, so that the exciting possibility emerges that the nascent science of $\mathrm{X}$-ray astronomy might provide important data for astronomers studying neutron star structure and pulsar mechanisms.

Actually, the idea of searching for $\mathrm{X}$-rays from neutron stars is not new. Before the appearance of pulsar astronomy, it remained the best hope for the eventual discovery of these strange objects. The excitement generated during the past few years by the spectacular pulsars has, however, quite eclipsed the consideration of $\mathrm{X}$-ray detection of neutron stars, and the subject makes a new appearance in the August 9 issue of Science (185, $487-490 ; 1974)$. The resurrection is promoted by two American astronomers, Greenstein of Amherst College Massachusetts, and McClintock of MIT. These authors point out that this $X$ radiation bears the same relation to neutron stars as continuum starlight does to ordinary stars. And where would astronomy be without starlight?

Recently, strenuous efforts have been made to construct theoretical models of neutron stars. The problems involved are formidable, for in almost every respect these objects represent matter in an extreme condition. Indeed, the entire neutron star may be envisaged as a sort of gigantic atomic nucleus. A correct understanding of such a strange object requires advances in several separate branches of physics. Consequently, a direct observation of neutron star radiation would provide the only source of observational support for theories of matter in this high density condition.

Specifically, Greenstein and McClintock suggest five pieces of information which could be deduced from a careful measurement of X-ray spectrum. The speotrum itself would give the surface temperature of the star and hence, knowing the total flux, its tadius could then be estimated, thereby confirming (or not) that the source really is a highly compact neutron star. In turn the radius could be used to determine the mass and the pulsar magnetic field, at least approximately. In addition, a knowledge of the mag. netic field, temperature and mass enables the age of the neutron star to be calculated, with the possibility of a fruitful comparison with the associated pulsar age which may be deduced from the rate of slowing down of the pulsar period. Finally, it is in principle possible to determine the internal temperature of the star, with a possible cross check here too from the details of the quasiexponential period decay which has been observed to occur after peculiar period jumps in the radiation pulses.

In their article, Greenstein and McClintock outline three theoretical procedures which may be used to obtain an estimate of the temperatures of neutron stars. The first is simply to try to calculate the rate of cooling as from the time of formation. The second is to examine various dissipative processes which generate heat inside the object as it rotates. In both these methods the condition of the relevant theoretical models does not inspire much confidence in the result, so that a crude upper limit may be placed on the temperature by summing the total available energy. Turning to the observational aspects of the subject, the authors paint a rather pessimistic picture. There is no observational evidence at all yet for thermal $\mathbf{X}$ rays from neutron stars. The best potential candidates are thought to be the middle aged pulsars-perhaps 100,000 years old -whose direct radiation would not be swamped by the pulsar radiation from the magnetosphere. From the present non-detection of $X$ rays from the known pulsars by the Uhuru X-ray observatory, upper limits on the temperature of 21 nearby pulsars are quoted. A comparison of a sample of these limits with the theoretical values obtained using the three methods described above is presented. Unfontunately, in all cases the theoretical values lie well below the observed upper limits, so that little of value may be gleaned from this.

Despite the rather discouraging situation so far, Greenstein and McClintock argue their case well, and it would be surprising if further observational and theoretical work were not initiated on this difficult but important topic for astronomers. And in view of the fact that the neutron star represents the most extreme density of matter in the observable Universe (greater densities lead to unobservable black hole matter) there is also a general scientific interest in their direct observation.

\section{P. C. W. Davies}

\section{Checking on laser fusion}

\section{from a Correspondent}

IN May this year, KMS Fusion Inc. claimed to have produced, for the first time in the United States, thermonuclear neutrons from a laser-compressed deuterated target. (The background to the laser-compression approach to fusion is outlined in Nature, 239, 139 and $129 ; 1972$.) K. M. Siegel, chairman of the company, later amplified some of these claims, which are the outcome of a private corporate investment which now amounts to $\$ 17.5$ million, in a talk given at Harwell on July 8.

Between May 1 and July 8, about 35 neutron-producing shots on deuterated and D-T targets had been made, of which $80 \%$ were successful. The prompt neutron yield (measured with a time resolution of $2 \mathrm{~ns}$ ) was as high as $5 \times 10^{5}$ when the target chamber was evacuated; the total yield increased to $1.8 \times 10^{7}$ when deuterium gas was admitted to the target chamber. (The latter result duplicated earlier Russian yields, in which neutron time-of-flight measurements were made with a detector resolution of $5 \mathrm{~ns}$ over flight paths of more than $3 \mathrm{~m}$.) X-ray pinhole photographs, with a spatial resolution of $5 \mu \mathrm{m}$, demonstrated that the implosion and compression of a hollow target was spherically symmetric. Time-resolved X-ray emission measurements suggested that a density increase of 80 times occurred when two opposing Nd-laser beams (total energy $<100 \mathrm{~J}$ ) were focused by ellipsoidal mirrors on to the (suspended) solid state target. 